\title{
Erratum to: The effect of fetal rat brain extract on morphology of bone marrow-derived mesenchymal stem cells
}

\author{
Iman Razeghian Jahromi ${ }^{1}$ - Davood Mehrabani ${ }^{2}$ Ali Mohammadi ${ }^{1}$. \\ Mehdi Dianatpour ${ }^{2,3}$ - Amin Tamadon ${ }^{2}$ - Shahrokh Zare ${ }^{2}$. \\ Mohammad M. Ghahramani Seno ${ }^{4}$ - Zahra Khodabandeh ${ }^{2}$
}

Published online: 28 September 2016

(C) Springer-Verlag London 2016

Erratum to: Comparative Clinical Pathology 25(2): 343-349

DOI 10.1007/s00580-015-2188-7

The original version of this article contained a mistake in one of the author name. Mehdi Ghahremani Seno should have been Mohammad M. Ghahramani Seno.

The online version of the original article can be found at http://dx.doi. org/10.1007/s00580-015-2188-7.

Davood Mehrabani

mehrabad@sums.ac.ir

Division of Biotechnology, Department of Pathobiology, School of Veterinary Medicine, Shiraz University, Shiraz, Iran

2 Stem Cell and Transgenic Technology Research Center, Shiraz University of Medical Science, Shiraz, Iran

3 Department of Human Genetic, School of Medicine, Shiraz University of Medical Sciences, Shiraz, Iran

4 Department of Basic Sciences and Division of Biotechnology, Department of Pathobiology, School of Veterinary Medicine, Ferdowsi University of Mashhad, Mashhad, Iran 